\title{
ChemComm
}

\section{Bottom-up solution synthesis of narrow nitrogen-doped graphene nanoribbons $\dagger$}

\begin{abstract}
Cite this: Chem. Commun., 2014, 50, 4172

Received 3rd February 2014 Accepted 5th March 2014

DOI: $10.1039 / \mathrm{c} 4 \mathrm{cc} 00885 \mathrm{e}$

www.rsc.org/chemcomm

Large quantities of narrow graphene nanoribbons with edgeincorporated nitrogen atoms can be synthesized via Yamamoto coupling of molecular precursors containing nitrogen atoms followed by cyclodehydrogenation using Scholl reaction.
\end{abstract}

\author{
Timothy H. Vo, ${ }^{a}$ Mikhail Shekhirev, ${ }^{a}$ Donna A. Kunkel, ${ }^{b}$ François Orange, \\ Maxime J.-F. Guinel, ${ }^{c d}$ Axel Enders ${ }^{\text {be }}$ and Alexander Sinitskii*ae
}

Graphene nanoribbons (GNRs), narrow strips of graphene with high aspect ratios, attract a great deal of attention because of their interesting electronic and magnetic properties. ${ }^{1}$ According to the theoretical studies, these properties strongly depend on the width of GNRs as well as their edge structure and termination, and are also very sensitive to the edge disorder. ${ }^{2-5}$ Thus, it is very important to precisely control the structure of GNRs on the atomic scale. Numerous top-down fabrication approaches have been developed to produce GNRs from graphite, graphene or carbon nanotubes, as discussed in the recent review papers, ${ }^{6-8}$ but the resulting ribbons typically have relatively large and non-uniform widths and disordered edges. Narrow atomically precise GNRs could be synthesized via bottom-up approaches that employ decomposition of molecules inside carbon nanotubes, ${ }^{9,10}$ or rely on the surfaceassisted $^{11-14}$ or solution polymerization ${ }^{8,15-22}$ of molecular precursors followed by cyclodehydrogenation of the resulting polymers. While surface-assisted bottom-up synthesis of GNRs yields small quantities of ribbons on metallic substrates in ultrahigh vacuum conditions that are ideal for scanning tunnelling microscopy (STM) characterization, solution-based approaches are preferred for the bulk synthesis of GNRs, so these two groups of methods could be considered as complimentary.

\footnotetext{
${ }^{a}$ Department of Chemistry, University of Nebraska - Lincoln, Lincoln, NE 68588, USA. E-mail: sinitskii@unl.edu

${ }^{b}$ Department of Physics, University of Nebraska - Lincoln, Lincoln, NE 68588, USA

${ }^{c}$ Department of Physics and Nanoscopy Facility, University of Puerto Rico,

PO Box 70377, San Juan, Puerto Rico 00936-8377, USA

${ }^{d}$ Department of Chemistry, University of Puerto Rico, PO Box 70377, San Juan, Puerto Rico 00936-8377, USA

${ }^{e}$ Nebraska Center for Materials and Nanoscience, University of Nebraska - Lincoln, Lincoln, NE 68588, USA

$\dagger$ Electronic supplementary information (ESI) available: Experimental details (synthesis and characterization). See DOI: 10.1039/c4cc00885e
}

Electronic properties of GNRs could be further tuned via their doping with heteroatoms, such as boron or nitrogen. ${ }^{23}$ This possibility has been extensively studied theoretically, ${ }^{24-30}$ but only a few experimental attempts to synthesize nitrogen-doped GNRs (N-GNRs) by bottom-up approaches have been reported. ${ }^{14,20}$ Bronner et al. prepared chevron-like N-GNRs by the surface-assisted approach on $\mathrm{Au}(111)$ substrate and performed their spectroscopic characterization. ${ }^{14}$ Kim et al. synthesized N-GNRs with a different structure in solution via Suzuki coupling of molecular precursors, one of which was dibrominated pyrazine, and cyclodehydrogenation of the resulting polymers. ${ }^{20}$ Here we demonstrate that highquality chevron-like N-GNRs could be synthesized via Yamamoto coupling of molecular precursors containing nitrogen atoms followed by cyclodehydrogenation via Scholl reaction (Fig. 1).

The structure of the N-GNR synthesized in this work is shown in Fig. 1a. Since the unit cell of this ribbon contains four nitrogen atoms, we refer to it as " $4 \mathrm{~N}-\mathrm{GNR}$ ”. The monomer molecule 3 used as a precursor for the $4 \mathrm{~N}-\mathrm{GNR}$ synthesis is not symmetric relative to the plane perpendicular to the direction

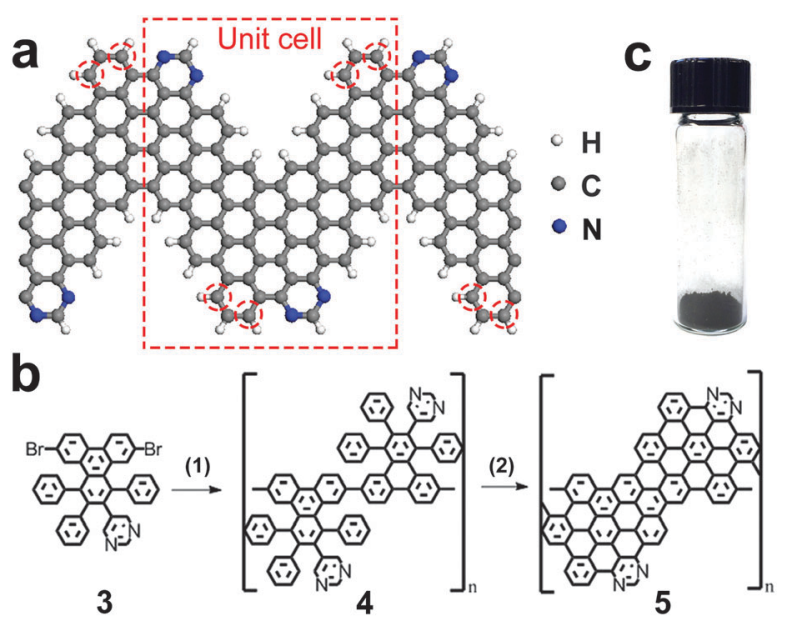

Fig. 1 Synthesis of 4N-GNRs. (a) Schematic of 4N-GNR. (b) Reaction scheme used in this work: (1) Yamamoto coupling, (2) Scholl reaction; see text and $\mathrm{ESI} \dagger$ for details. (c) Optical photograph of a $4 \mathrm{~N}-\mathrm{GNR}$ powder. 
of coupling (Fig. 1b), so as the result, the positions of nitrogen atoms in the ribbon edge could vary, i.e. the nitrogens could also occupy the positions highlighted with red circles in Fig. 1a instead of the ones shown in the schematic. However, the $\mathrm{N}: \mathrm{C}$ ratio of $1: 20$ in this ribbon should be the same regardless of how the monomer molecules couple with each other.

The synthesis and characterization of the precursor molecule 3, as well as the reactions used to convert monomer 3 to polymer 4 to 4N-GNR 5 (Fig. 1b) are described in detail in the ESI. $\dagger$ About $100 \mathrm{mg}$ of $4 \mathrm{~N}-\mathrm{GNRs}$ was obtained in a single reaction (Fig. 1c). Previously, we have demonstrated that a similar procedure could be used to obtain $>1 \mathrm{~g}$ of chevron-type GNRs (nitrogen-free analogues of $4 \mathrm{~N}-\mathrm{GNRs}$ shown in Fig. 1a) in a single reaction, ${ }^{21}$ so we believe that the synthesis of $4 \mathrm{~N}-$ GNRs could also be scaled up to at least a gram scale. Though not soluble in any solvents that we tried, these ribbons could be suspended by sonication in, for example, toluene or mesitylene, and then deposited on different substrates for microscopic and spectroscopic characterization.

It was previously demonstrated that when chevron-type GNRs are deposited from dispersion to a substrate, they could be found in a form of elongated self-assembled nanostructures, referred to as "GNR nanobelts". 21 These nanobelts are only one carbon atom thick and comprise multiple GNRs arranged in a side-by-side fashion. ${ }^{21}$ We deposited $4 \mathrm{~N}$-GNRs on a variety of substrates (mica, Au(111), copper TEM grid with ultrathin carbon support film) and observed similar nanobelts. Fig. 2a shows an atomic force microscopy (AFM)

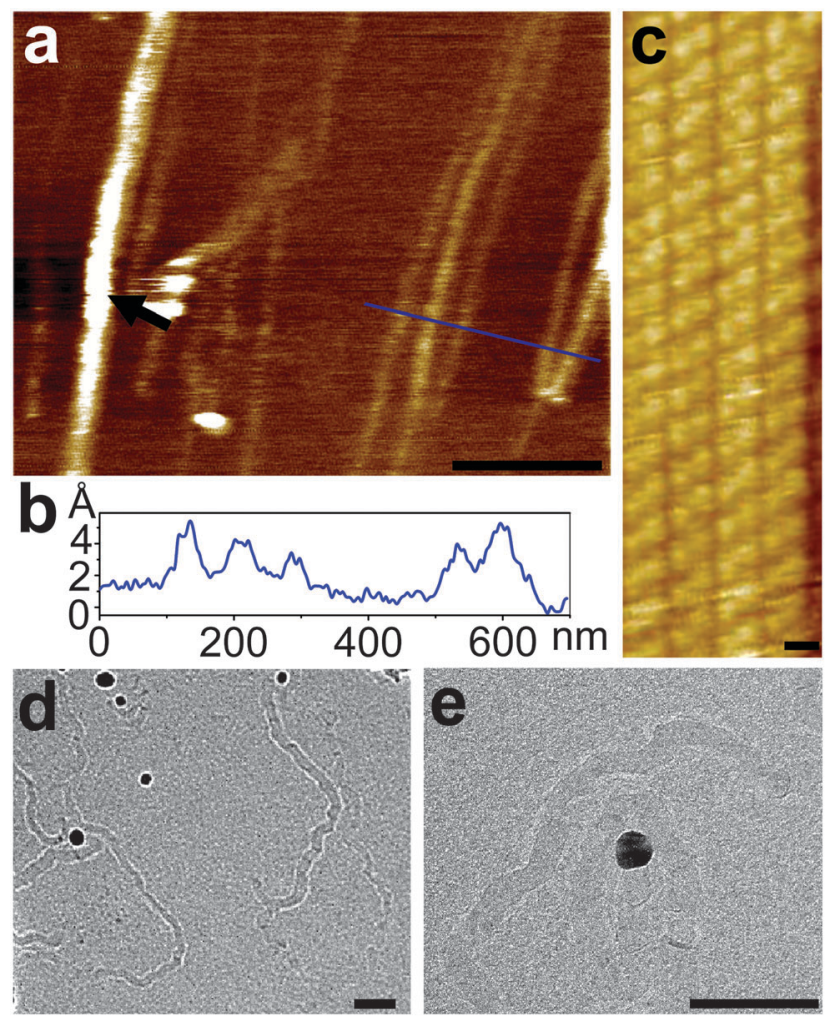

Fig. 2 Microscopic characterization of 4N-GNRs. (a) AFM image of 4N-GNRs deposited on mica. Scale bar is $400 \mathrm{~nm}$. (b) Height profile along the blue line shown in (a). (c) STM image of $4 \mathrm{~N}$-GNRs deposited on a Au(111) single crystal. Scale bar is $1 \mathrm{~nm}$. (d, e) TEM images of $4 \mathrm{~N}-$ GNRs. Scale bars are $50 \mathrm{~nm}$. image of $4 \mathrm{~N}-\mathrm{GNRs}$ that were dispersed in toluene by sonication, deposited on freshly cleaved mica and dried prior to imaging. Observed in this image are high-aspect-ratio nanobelts that are several $\mu \mathrm{m}$ long. The alignment of $4 \mathrm{~N}-\mathrm{GNR}$ nanobelts likely happens in the contact angle between a droplet of $4 \mathrm{~N}-\mathrm{GNR}$ dispersion and a substrate during the solvent drying. As shown in the height profile measured along the blue line in Fig. 2a, the majority of these nanobelts have heights $<5 \AA$, which corresponds to the thickness of a single layer or graphene (Fig. 2b). In fact, in multiple AFM images we observed only one structure with a thickness $(t)$ larger than $5 \AA$, which is indicated by the black arrow in Fig. 2a. This structure $(t \sim 1 \mathrm{~nm})$ is likely a rare stack of $2-3$ nanobelts. Fig. 2c shows an STM image of the fragment of a $4 \mathrm{~N}-\mathrm{GNR}$ nanobelt on $\mathrm{Au}(111)$, which illustrates the side-by-side arrangement of individual ribbons that are over $40 \mathrm{~nm}$ long. As we indicated in the previous study of solution-synthesized chevron-like GNRs, it remains unclear if these nanobelts exist in solution or form directly on a substrate by capillary forces during the solvent evaporation. ${ }^{21} 4 \mathrm{~N}-\mathrm{GNR}$ nanobelts were also imaged by transmission electron microscopy (TEM), see Fig. $2 \mathrm{~d}$ and e. The nanobelts found on the TEM grids are 8 to $20 \mathrm{~nm}$ wide, which corresponds to 8-12 4N-GNRs arranged in a side-by-side fashion, and up to $1 \mu \mathrm{m}$ long.

Raman spectroscopy is very sensitive to the disorder (edges and other structural defects, chemical functionalization, etc.) in carbon materials, ${ }^{31}$ so we used this method to confirm the high structural quality of $4 \mathrm{~N}-$ GNRs. Fig. 3 shows the Raman spectrum of $4 \mathrm{~N}-\mathrm{GNRs}$, where in addition to the most intense lines at 1324 and $1600 \mathrm{~cm}^{-1}$ that are typically referred to as D and $\mathrm{G}$ bands, respectively, ${ }^{31}$ a series of smaller peaks around the D and $G$ lines could be observed. This fine structure is a direct result of the low dimensionality of a nanoribbon, and the relative intensities and positions of the lines are characteristic for each specific polycyclic aromatic hydrocarbon (PAH). ${ }^{32} 4 \mathrm{~N}$-GNRs synthesized in this work are structurally close to chevron-like GNRs, so this is not surprising that the Raman spectrum of $4 \mathrm{~N}-\mathrm{GNRs}$ is very similar to that of chevron-like GNRs synthesized by the solution approach. ${ }^{21}$ Since disordered carbon materials usually show only broad D and $\mathrm{G}$ lines without any fine features, the spectrum shown in Fig. 3 indicates the high structural quality of $4 \mathrm{~N}-\mathrm{GNRs}$.

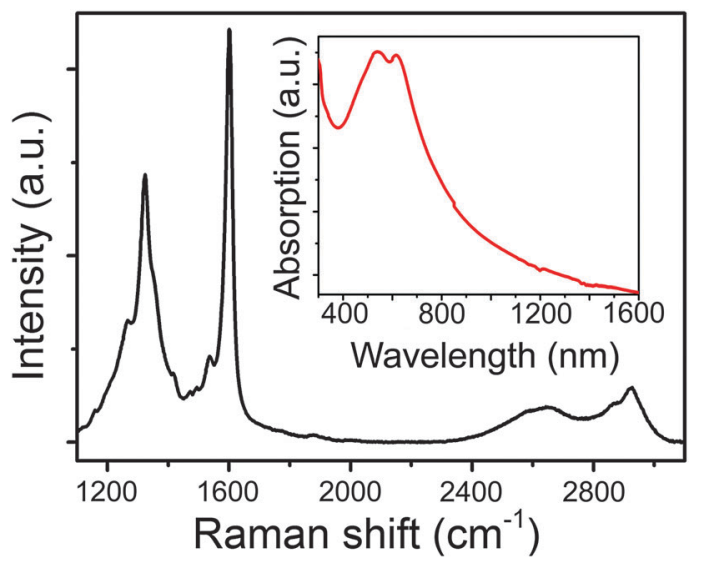

Fig. 3 Raman spectrum of $4 N-G N R s$. The inset shows UV-vis-NIR absorption spectrum of $4 \mathrm{~N}$-GNRs suspended in mesitylene by sonication. 

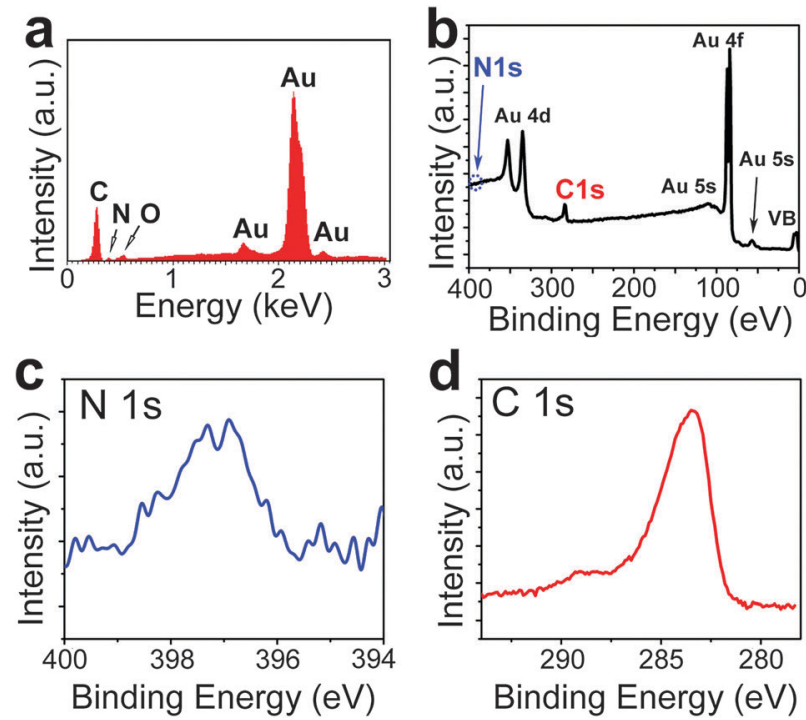

Fig. 4 Spectroscopic characterization of $4 N-G N R s$. (a) EDX spectrum of $4 N-G N R s$ deposited on a gold substrate. (b-d) XPS spectra of $4 N-G N R s$ deposited on a gold substrate: (b) survey spectrum, (c) N 1s spectrum and (d) C 1s spectrum.

The inset in Fig. 3 shows a UV-vis-NIR absorption spectrum of $4 \mathrm{~N}-$ GNRs that were suspended in mesitylene by sonication. This spectrum is qualitatively similar to the UV-vis-NIR spectra of other solution-synthesized GNRs, showing a strong absorption in the visible range that gradually decreases with the wavelength increasing toward the infrared region. ${ }^{19,21}$ The optical band gap of $4 \mathrm{~N}-\mathrm{GNRs}$ determined from this spectrum is $\sim 1.6 \mathrm{eV}$.

In order to confirm the presence of nitrogen atoms in the structure of $4 \mathrm{~N}-\mathrm{GNRS}$ we characterized the ribbons by energydispersive X-ray (EDX) spectroscopy and X-ray photoelectron spectroscopy (XPS). Fig. 4a shows an EDX spectrum of 4N-GNRs deposited on a gold foil. The nitrogen peak is detectable although small, which is not surprising, considering the expected $\mathrm{N}: \mathrm{C}$ ratio of $1: 20$. The only foreign peak observed in the spectrum is the low intensity $\mathrm{O}$ line, which is likely caused by atmospheric adsorbates. The XPS survey spectrum (Fig. 4b) of the same sample demonstrates only the peaks associated with the GNRs and the gold substrate. XPS N 1s spectrum of 4N-GNRs (Fig. 4c) shows a single peak at $397.1 \mathrm{eV}$, which is close to pyridinic nitrogen peaks in $\mathrm{N}$-doped graphene. ${ }^{33}$ XPS C 1s spectrum (Fig. 4d) contains the main peak corresponding to the $\mathrm{C}-\mathrm{C}$ bonds and the weaker peak at $\sim 288.5$ eV likely representing $\mathrm{C}-\mathrm{N}$ bonds. ${ }^{33,34}$

In summary, we demonstrate that the synthetic approach based on Yamamoto coupling of $\mathrm{N}$-doped molecular precursors and cyclodehydrogenation via Scholl reaction could be used to synthesize high-quality $4 \mathrm{~N}-\mathrm{GNRs}$. The ribbons were investigated by the combination of microscopic (STM, AFM, TEM) and spectroscopic (UV-vis-NIR, XPS, EDX and Raman spectroscopy) techniques. In the future studies, we will investigate if this approach could also be employed for the synthesis of N-GNRs with other structures and $\mathrm{N}: \mathrm{C}$ ratios.

This work was supported by the Nebraska Center for Energy Sciences Research (\#12-00-13), the Nebraska Research Initiative and the NSF through Nebraska MRSEC (DMR-0820521) and EPSCoR (EPS-1004094).

\section{Notes and references}

1 O. V. Yazyev, Acc. Chem. Res., 2013, 46, 2319-2328.

2 L. Yang, C.-H. Park, Y.-W. Son, M. L. Cohen and S. G. Louie, Phys. Rev. Lett., 2007, 99, 186801.

3 V. Barone, O. Hod and G. E. Scuseria, Nano Lett., 2006, 6, 2748-2754.

4 D. A. Areshkin, D. Gunlycke and C. T. White, Nano Lett., 2006, 7, 204-210.

5 E. R. Mucciolo, A. H. Castro Neto and C. H. Lewenkopf, Phys. Rev. B, 2009, 79, 075407.

6 M. Terrones, A. R. Botello-Méndez, J. Campos-Delgado, F. LópezUrías, Y. I. Vega-Cantú, F. J. Rodríguez-Macías, A. L. Elías, E. MuñozSandoval, A. G. Cano-Márquez, J.-C. Charlier and H. Terrones, Nano Today, 2010, 5, 351-372.

7 L. Ma, J. Wang and F. Ding, ChemPhysChem, 2013, 14, 47-54.

8 L. Chen, Y. Hernandez, X. L. Feng and K. Mullen, Angew. Chem., Int. Ed., 2012, 51, 7640-7654.

9 A. Chuvilin, E. Bichoutskaia, M. C. Gimenez-Lopez, T. W. Chamberlain, G. A. Rance, N. Kuganathan, J. Biskupek, U. Kaiser and A. N. Khlobystov, Nat. Mater., 2011, 10, 687-692.

10 T. W. Chamberlain, J. Biskupek, G. A. Rance, A. Chuvilin, T. J. Alexander, E. Bichoutskaia, U. Kaiser and A. N. Khlobystov, ACS Nano, 2012, 6, 3943-3953.

11 J. M. Cai, P. Ruffieux, R. Jaafar, M. Bieri, T. Braun, S. Blankenburg, M. Muoth, A. P. Seitsonen, M. Saleh, X. L. Feng, K. Mullen and R. Fasel, Nature, 2010, 466, 470-473.

12 S. Blankenburg, J. M. Cai, P. Ruffieux, R. Jaafar, D. Passerone, X. L. Feng, K. Mullen, R. Fasel and C. A. Pignedoli, ACS Nano, 2012, 6, 2020-2025.

13 Y.-C. Chen, D. G. de Oteyza, Z. Pedramrazi, C. Chen, F. R. Fischer and M. F. Crommie, ACS Nano, 2013, 7, 6123-6128.

14 C. Bronner, S. Stremlau, M. Gille, F. Brauße, A. Haase, S. Hecht and P. Tegeder, Angew. Chem., Int. Ed., 2013, 52, 4422-4425.

15 J. Sakamoto, J. van Heijst, O. Lukin and A. D. Schluter, Angew. Chem., Int. Ed., 2009, 48, 1030-1069.

16 X. Y. Yang, X. Dou, A. Rouhanipour, L. J. Zhi, H. J. Rader and K. Mullen, J. Am. Chem. Soc., 2008, 130, 4216-4217.

17 Y. Fogel, L. Zhi, A. Rouhanipour, D. Andrienko, H. J. Räder and K. Müllen, Macromolecules, 2009, 42, 6878-6884.

18 L. Dossel, L. Gherghel, X. L. Feng and K. Mullen, Angew. Chem., Int. Ed., 2011, 50, 2540-2543.

19 M. G. Schwab, A. Narita, Y. Hernandez, T. Balandina, K. S. Mali, S. De Feyter, X. L. Feng and K. Mullen, J. Am. Chem. Soc., 2012, 134, 18169-18172.

20 K. T. Kim, J. W. Lee and W. H. Jo, Macromol. Chem. Phys., 2013, 214, 2768-2773.

21 T. H. Vo, M. Shekhirev, D. A. Kunkel, M. D. Morton, E. Berglund, L. M. Kong, P. M. Wilson, P. A. Dowben, A. Enders and A. Sinitskii, Nat. Commun., 2014, 5, 3189.

22 A. Narita, X. Feng, Y. Hernandez, S. A. Jensen, M. Bonn, H. Yang, I. A. Verzhbitskiy, C. Casiraghi, M. R. Hansen, A. H. R. Koch, G. Fytas, O. Ivasenko, B. Li, K. S. Mali, T. Balandina, S. Mahesh, S. De Feyter and K. Mullen, Nat. Chem., 2014, 6, 126-132.

23 R. Lv and M. Terrones, Mater. Lett., 2012, 78, 209-218.

24 F. Cervantes-Sodi, G. Csányi, S. Piscanec and A. C. Ferrari, Phys. Rev. B, 2008, 77, 165427.

25 S. S. Yu, W. T. Zheng, Q. B. Wen and Q. Jiang, Carbon, 2008, 46, 537-543.

26 Y. Li, Z. Zhou, P. Shen and Z. Chen, ACS Nano, 2009, 3, 1952-1958.

27 X.-L. Wei, H. Fang, R.-Z. Wang, Y.-P. Chen and J.-X. Zhong, Appl. Phys. Lett., 2011, 99, 012107.

28 H. Kim, K. Lee, S. I. Woo and Y. Jung, Phys. Chem. Chem. Phys., 2011, 13, 17505-17510.

29 E. Cruz-Silva, Z. M. Barnett, B. G. Sumpter and V. Meunier, Phys. Rev. $B, 2011,83,155445$.

30 X. H. Zheng, X. L. Wang, T. A. Abtew and Z. Zeng, J. Phys. Chem. C, 2010, 114, 4190-4193.

31 A. C. Ferrari and D. M. Basko, Nat. Nanotechnol., 2013, 8, 235-246.

32 F. Negri, C. Castiglioni, M. Tommasini and G. Zerbi, J. Phys. Chem. A, 2002, 106, 3306-3317.

33 D. Wei, Y. Liu, Y. Wang, H. Zhang, L. Huang and G. Yu, Nano Lett., 2009, 9, 1752-1758.

34 A. P. Dementjev, A. de Graaf, M. C. M. van de Sanden, K. I. Maslakov, A. V. Naumkin and A. A. Serov, Diamond Relat. Mater., 2000, 9, 1904-1907. 